\title{
Supplementary Information \\ Quantitative determination of the complex polarizability of individual nanoparticles by scanning cavity microscopy
}

\author{
Matthias Mader, Julia Benedikter, Lukas Husel, \\ Theodor W. Hänsch, David Hunger
}

\section{Supplementary Notes}

$1 \quad$ Extinction Cross Section and Cavity Losses $\quad$ S1

2 Optical Properties of a Metal Nanoparticle in the Rayleigh Limit S3

3 Repeatability of Extinction Measurements based on Scanning Cavity Microscopy S5

\begin{tabular}{ll}
\hline Supplementary References & S7
\end{tabular}

\section{Supplementary Note 1: Extinction Cross Section and Cavity Losses}

The transmission $T_{c a v}$ on resonance and the linewidth $\kappa$ of the resonance of an optical resonator is determined on the one hand by the transmission $T_{i}$ and the losses $L_{i}$ of the two mirrors $i=1,2$ and on the other hand by the losses due to the medium $L$, the light passes within the resonator. Here $L$ denotes the portion of light getting lost when passing the medium once. The maximum transmission is given by

$$
T_{c a v}=\frac{4 T_{1} T_{2}}{\left(2 L+L_{1}+L_{2}+T_{1}+T_{2}\right)^{2}},
$$

the linewidth by

$$
\kappa=\frac{c}{2 \pi d} \frac{2 L+L_{1}+L_{2}+T_{1}+T_{2}}{2}
$$

where $c$ is the speed of light and $d=q \lambda / 2$ the cavity length with the wavelength $\lambda$ and the longitudinal mode order $q$.

For the losses $L$ inside a resonator, no further assumptions concerning position or shape of the lossy medium are neither made nor necessary.

In contrast, the loss of light due to a nanoparticle is described by its extinction cross section $\sigma_{\text {ext }}$. This quantity associates a perfectly absorbing disk of a certain size to the particle, blocking the light passing it. 
To bring both concepts together, the extinction cross section has to be compared to the mode size of the light field in the resonator. Before doing so, let's consider the problem in free space, where a Gaussian beam with waist $w_{0}$ gets attenuated by a nanoparticle in its center that has an extinction cross section much smaller than the beam size.

A Gaussian beam has an intensity distribution of

$$
I(r)=I_{0} \mathrm{e}^{-\frac{2 r^{2}}{w_{0}^{2}}}
$$

where $I_{0}$ is the peak intensity and $w_{0}$ the $1 / \mathrm{e}^{2}$-radius of the Gaussian. Integrating over the full area yields a relation between the peak intensity $I_{0}$ and the total power of the beam $P_{0}$, that can be measured:

$$
P_{0}=\int_{0}^{\infty} \int_{0}^{2 \pi} I_{0} \mathrm{e}^{-\frac{2 r^{2}}{w_{0}^{2}}} r \mathrm{~d} \varphi \mathrm{d} r=I_{0} \frac{\pi w_{0}^{2}}{2} .
$$

If a small absorbing disk with radius $r_{\text {ext }}$ and surface area $\sigma_{\text {ext }}$ is brought to the center of the beam, the absorbed power $P_{L}$ can be determined by

$$
P_{L}=\int_{0}^{r_{e x t}} \int_{0}^{2 \pi} I_{0} \mathrm{e}^{-\frac{2 r^{2}}{w_{0}^{2}}} r \mathrm{~d} \varphi \mathrm{d} r
$$

as $r_{\text {ext }}$ is assumed to be much smaller than $w_{0}$, the radial dependence of the intensity can be neglected and the integral simplifies to

$$
P_{L} \approx I_{0} \int_{0}^{r_{e x t}} \int_{0}^{2 \pi} r \mathrm{~d} \varphi \mathrm{d} r=I_{0} r_{e x t}^{2} \pi=I_{0} \sigma_{e x t} .
$$

The fraction of the light $L$, getting lost at the absorbing disk that can be associated with the extinction cross section of a nanoparticle is determined by comparing the absorbed power to the incident power:

$$
L=\frac{P_{L}}{P_{0}}=\frac{I_{0} \sigma_{e x t}}{I_{0} \frac{\pi w_{0}^{2}}{2}}=\frac{2 \sigma_{e x t}}{\pi w_{0}^{2}} .
$$

In contrast to the free space problem, where a running wave interacts with the particle and a spatial as well as a temporal average of the light wave has to be considered, in a resonator, a standing wave builds up. Thus the position of the particle in the standing wave plays an important role. If placed to an antinode of the wave, as done in the experiments presented in this work, the intensity here is a factor of 2 higher compared to the spatial average of the running wave case, the extinction cross section is defined for. Thus the losses at the particle get modified to

$$
L=\frac{4 \sigma_{e x t}}{\pi w_{0}^{2}} .
$$

In the context of quantum optics, this increase of losses due to an absorbing particle inside an optical cavity is extensively discussed e.g. by Tanji-Suzuki et. al. 1 ] 


\section{Supplementary Note 2: Optical Properties of a Metal Nanopar- ticle in the Rayleigh Limit}

For the interpretation of the experiments shown in this work, a detailed understanding of the optical properties of the metal nanoparticles is crucial. As the particles are small compared to the wavelength $\lambda\left(r_{\text {part }} \ll \lambda /\left(2 \pi n_{\text {env }}\right)\right)$ it is possible to simplify the problem to the treatment introduced by Lord Rayleigh. In the following, different effects that influence the properties are discussed.

The dielectric constants of the particle, its surrounding and the surfaces in the vicinity of the particle are denoted as follows: $\epsilon_{1}$ denotes the dielectric constant of the surrounding environment, $\epsilon_{2}$ the dielectric constant of a surface close to the particle and $\epsilon_{3}$ gives the dielectric constant of the particle itself as depicted in figure S1 $\epsilon_{0}$ is the vacuum dielectric constant.

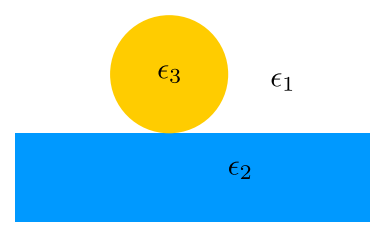

Supplementary Figure S1: Optical constants of the particle, the surface it is placed to and it's environment

\section{Polarizability of a spherical nanoparticle}

The polarizability is the key quantity to describe the optical properties of a particle. For spherically symmetric and isotropic particles, the polarizability is given by the Clausius-Mossotti law:

$$
\alpha=3 \epsilon_{0} V_{p} \frac{\epsilon_{3}-\epsilon_{1}}{\epsilon_{3}+2 \epsilon_{1}}
$$

where $V_{p}=4 \pi r^{3} / 3$ is the volume of the particle.

\section{Correction of the dielectric constant because of the small size}

It turns out that the dielectric constant of gold $\epsilon_{3}$ has to be modified with respect to the bulk value $\left(\epsilon_{b u l k}\right)^{2}$ in order to describe very small particles accurately ${ }^{3}$ :

$$
\epsilon_{3}(\lambda)=\epsilon_{\text {bulk }}(\lambda)+\frac{\omega_{p}^{2}}{\omega\left(\omega+i \gamma_{b u l k}\right)}-\frac{\omega_{p}^{2}}{\omega(\omega+i \gamma)},
$$

with the metal plasma frequency $\omega_{p}$ and the electron scattering rates in the bulk $\left(\gamma_{\text {bulk }}\right)$ and confinded metal $(\gamma)$, where

$$
\gamma=\gamma_{b u l k}+2 g v_{F} / D
$$

with $v_{F}$ the Fermi velocity, $D$ the particle diameter and $g$ a proportionality factor in the order of 1 . 


\section{Correction of the polarizability for particles on a dielectric sur- face}

As the nanoparticles in our experiments lie on the surface of a dielectric mirror, also the effect of a surface close to the particle has to be considered. For metal nanospheres far away from plasmon resonance and for an incident light field with a polarization parallel to the surface, like in the case discussed in this paper, Wind et al. $\frac{45}{6}$ give the following solution:

$$
\alpha=\epsilon_{0} \frac{\epsilon_{1}\left(\epsilon_{3}-\epsilon_{1}\right) V_{p}}{\epsilon_{1}+L_{s}\left(\epsilon_{3}-\epsilon_{1}\right)}
$$

with the depolarization factor

$$
L_{s}=\frac{1}{3}\left(1-\frac{1}{8} \frac{\epsilon_{2}-\epsilon_{1}}{\epsilon_{2}+\epsilon_{1}}\right) .
$$

\section{Scattering and Absorption}

When light interacts with an object it either gets absorbed or scattered. For nanoparticles smaller than a wavelength of light, this is described by Rayleigh scattering. The corresponding cross sections are given by ${ }^{6}$.

$$
\sigma_{a b s}=\frac{2 \pi \sqrt{\epsilon_{1}}}{\lambda \epsilon_{0}} \Im(\alpha)
$$

and

$$
\sigma_{s c a}=\left(\frac{2 \pi}{\lambda}\right)^{4} \frac{|\alpha|^{2}}{6 \pi \epsilon_{0}^{2}} .
$$

As both effects attenuate the probe beam, the measured quantity in all our experiments is the extinction of a nanoparticle:

$$
\sigma_{e x t}=\sigma_{a b s}+\sigma_{s c a}
$$

\section{Size- and g-factor distribution for gold nanospheres}

The size of the nanoparticles varies as well as the $g$-factor, a heuristic value characterizing damping effects for small nanoparticles. In order to compare our measured distribution of extinction cross sections, we take a Gaussian diameter distribution measured by the manufacturer of the particles with central diameter $48.5 \mathrm{~nm}$ and a standard deviation of $1.9 \mathrm{~nm}$. In addition, we adopt a gaussian distribution of the $g$-factor found by Muskens et. al. ${ }^{[3}$ around 1.4 with a standard deviation of 0.25 . 


\section{Supplementary Note 3: Repeatability of Extinction Measure- ments based on Scanning Cavity Microscopy}

We have investigated the repeatability of scanning cavity microscopy already in earlier experiments and show here briefly the central results.

In that experiment, we have measured the extinction cross section of polystyrene spheres with a diameter of $(100.0 \pm 12.1) \mathrm{nm}^{1}$ using scanning cavity microscopy. Although the sample is different to gold particles that have been used in the present main study, the underlying physics describing scattering and absorption in the Rayleigh-limit is the same for both types of particles. This shows that the technique also works for purely scattering particles.

In contrast to the method presented in this paper where the change of the linewidth of the resonator has been measured in order to determine the extinction cross section of the nanoparticles, here the peak transmission of the resonator has been analyzed. We have demonstrated earlier that both methods are equivalent ${ }^{7}$.

In a first step, we have measured 15 times the extinction cross section $\sigma_{\text {ext }}$ of the same polystyrene particle at a longitudinal mode order of $q=14$ at a wavelength of $785.46 \mathrm{~nm}$. Figure S2 shows the results for each measurement. We have calculated a mean extinction cross section of $(133.3 \pm 0.7) \mathrm{nm}^{2}$. The corresponding relative deviation of the measurement is $0.5 \%$, proofing the high repeatability and precision of the method.

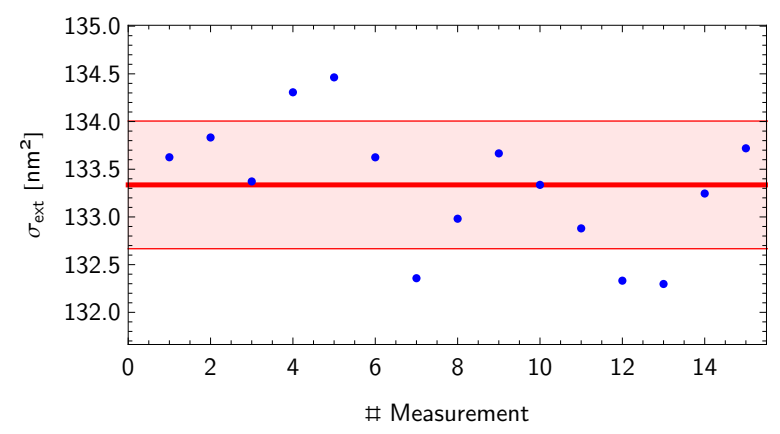

Supplementary Figure S2: Results of repeated measurements of the extinction cross section $\sigma_{\text {ext }}$ of a single polystyrene nano sphere at a longitudianl mode order of $q=14$. The mean value of $133.3 \mathrm{~nm}^{2}$ with its error band of $\pm 0.7 \mathrm{~nm}$ is depicted in red.

In a second step, we have measured the extinction cross section of the same particle for subsequent longitudinal mode orders and repeated this experiment 5 times. The average values of the measurement at each resonator length with its error bars is shown in figure $\mathrm{S3}$ Now larger variations between different longitudinal mode orders are visible, the mean value of the extinction cross section $\sigma_{\text {ext }}$ shifts to $(142 \pm$ $5) \mathrm{nm}^{2}$, the respective relative variation of the measurement is $5 \%$. This represents the leading systematic uncertainty which limits the accuracy of our method.

The variations are mainly caused by the coupling of higher order transversal modes to the fundamental cavity mode ${ }^{899}$ : in contrast to the wavefront of an ideal Gaussian mode, the profile especially of the mirror on the fiber has no spherical

\footnotetext{
${ }^{1}$ Polyscience Inc.
} 


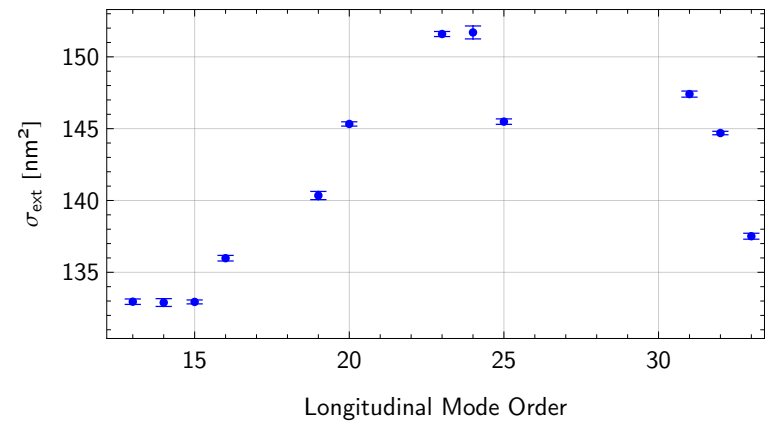

Supplementary Figure S3: Results of repeated measurements of the extinction cross section $\sigma_{e x t}$ of a single polystyrene nano sphere at subsequent logitudinal mode orders. Missing data points due to mode mixing.

but more a Gaussian profile 10111 . Thus the cavity mode is no longer an exact single Hermite-Gaussian mode but it can be described as a superposition of various Hermite-Gaussian modes, resembling a wavefront at the mirrors following their geometrical shape. At certain resonator lengths, a higher transversal mode of a subsequent longitudinal order has the same resonance frequency as the fundamental mode of the resonator, thus the modes couple and energy is transferred from one mode to the other. As this effect strongly depends on the exact geometry of both mirrors of the cavity, it can appear at a certain length where the finesse drops significantly and the cavity can not be used for scanning cavity microscopy. These longitudinal mode orders are not considered for measurements and correspond to the missing data points in figure 53 . The mode mixing effect can also have an effect for detuned transverse modes, however much less pronounced, but still leading to a slight variation of the measured extinction cross section for different longitudinal mode orders, as can be seen in figure $\mathrm{S3}$.

The measurements on polystyrene spheres presented in the section show that scanning cavity microscopy provides highly repeatable results. Nevertheless, the longitudinal mode order, the resonator is operated at has to be chosen carefully to avoid systematic errors especially due to mode mixing. 


\section{Supplementary References}

[1] Tanji-Suzuki, H.; Leroux, I. D.; Schleier-Smith, M. H.; Cetina, M.; Grier, A. T.; Simon, J.; Vuletić, V. Advances In Atomic, Molecular, and Optical Physics; Elsevier, 2011; pp 201-237.

[2] Johnson, P. B.; Christy, R. W. Optical Constants of the Noble Metals. Physical Review B 1972, 6, 4370-4379.

[3] Muskens, O. L.; Billaud, P.; Broyer, M.; Fatti, N. D.; Vallée, F. Optical extinction spectrum of a single metal nanoparticle: Quantitative characterization of a particle and of its local environment. Physical Review B 2008, 78, 205410.

[4] Wind, M. M.; Vlieger, J.; Bedeaux, D. The polarizability of a truncated sphere on a substrate I. Physica A: Statistical Mechanics and its Applications 1987, $141,33-57$.

[5] Wind, M.; Bobbert, P.; Vlieger, J.; Bedeaux, D. The polarizability of a truncated sphere on a substrate II. Physica A: Statistical Mechanics and its Applications 1987, 143, 164-182.

[6] van de Hulst, H. C. Light Scattering by Small Particles (Dover Books on Physics); Dover Publications, 1981.

[7] Mader, M.; Reichel, J.; Hänsch, T. W.; Hunger, D. A scanning cavity microscope. Nature Communications 2015, 6, 7249.

[8] Benedikter, J.; Hümmer, T.; Mader, M.; Schlederer, B.; Reichel, J.; Hänsch, T. W.; Hunger, D. Transverse-mode coupling and diffraction loss in tunable Fabry-Pérot microcavities. New Journal of Physics 2015, 17, 053051.

[9] Benedikter, J.; Moosmayer, T.; Mader, M.; Hümmer, T.; Hunger, D. Transverse-mode coupling effects in scanning cavity micrsocopy. New Journal of Physics 2019, 21, 103029.

[10] Hunger, D.; Steinmetz, T.; Colombe, Y.; Deutsch, C.; Hänsch, T. W.; Reichel, J. A fiber Fabry-Perot cavity with high finesse. New Journal of Physics 2010, 12, 065038.

[11] Hunger, D.; Deutsch, C.; Barbour, R. J.; Warburton, R. J.; Reichel, J. Laser micro-fabrication of concave, low-roughness features in silica. AIP Advances 2012, 2, 012119. 